\title{
Role of the CD62L Expression Pathway in the IVIg Inhibition of Cytotoxic T Cell Responses
}

Dominique Chabot ${ }^{1,2}$, Patrick Trépanier ${ }^{1,2}$, Cassandra Ringuette-Goulet ${ }^{1,3}$, Lionel Loubaki ${ }^{1}$ and Renée Bazin ${ }^{1,2 *}$

${ }^{1}$ Department of Research and Development, Héma-Québec, Québec, Canada

${ }^{2}$ Department of Biochemistry, Microbiology and Bioinformatics, Laval University, Québec, Canada

${ }^{3}$ Faculty of Pharmacy, Laval University, Québec, Canada

\begin{abstract}
Intravenous immunoglobulin (IVIg) is successfully used in the treatment of a number of immune-mediated disorders, including diseases in which self-reactive cytotoxic CD8+ T lymphocytes (CTLs) play an important pathological role. However, the exact molecular and cellular mechanisms underlying its effects on the cytotoxic response remain undefined. Using a mouse model of ovalbumin (OVA) immunization, we recently showed that IVIg treatment decreases the in vivo generation of OVA-specific CD8+ T cells, as well as the proportion of CTLs expressing the extracellular cytotoxic marker CD107a. In the present work, we demonstrate that during the course of an active immune response in mice, IVIg treatment is associated with the presence of splenic CD8+ T cells expressing high levels of CD62L and by an increased plasma concentration of soluble CD62L. Because cell surface expression of CD62L negatively correlates with the cytotoxic activity of CD8+ T cells and that soluble CD62L exhibits anti-inflammatory effects, we herein propose that the CD62L expression pathway plays a key role in the therapeutic effects of IVIg in CD8-mediated autoimmune and inflammatory disorders.
\end{abstract}

Keywords: Intravenous immunoglobulin; L-selectin; Cytotoxicity; Autoimmunity

\section{Introduction}

We recently showed, using a mouse model of ovalbumin (OVA) immunization, that intravenous immunoglobulin (IVIg) inhibits the cytotoxic CD8+ $\mathrm{T}$ lymphocyte (CTL) responses [1]. Indeed, IVIg injection significantly decreases the in vivo generation of OVAspecific CD8+ T cells as well as the proportion of CTLs expressing the cytotoxic-associated marker CD107a [1], which was shown to correlate with cytotoxic activities of CD8+ T cells [2]. However, the mechanisms underlying the effects of IVIg on the cytotoxic response remain unclear.

L-selectin, also known as CD62 ligand (CD62L), is an adhesion molecule mostly recognized for its role in lymphocyte homing and migration to lymphoid tissues and sites of inflammation [3,4]. CD62L is expressed on granulocytes, monocytes and lymphocytes, including CTLs [5]. A recent study using in vitro generated anti-tumor antigenspecific CD8+ $\mathrm{T}$ cells and melanoma lines as a model of activation revealed that $\mathrm{CD} 107 \mathrm{a}$ expression could only be detected on cells that had shed CD62L [6]. Conversely, CD8+ T cells engineered to express a shedding-resistant mutant of CD62L exhibited a reduced ability to lyse specific targets. These results led to the conclusion that there was a direct correlation between the loss of CD62L expression on CD8+ $\mathrm{T}$ cells and the acquisition of both cytotoxic activity and surface expression of CD107a [6]. We thus hypothesized that IVIg stimulates the expression of CD62L to modulate the CTL activity. The effect of IVIg on CD62L expression of splenic T cells was thus studied using the previously described mouse model of OVA immunization [1]. In addition, since soluble CD62L (sCD62L) is known to exhibit anti-inflammatory activities [7], we also determined its plasmatic concentration in IVIg-treated, OVA-immunized mice.

\section{Materials and Methods}

\section{Animals}

Wild-type female C57BL/6 mice (18-22 g) were obtained from Charles River (Montreal, Canada). Mice were kept at the animal facility at Laval University (Quebec City, Canada) and all procedures were approved by the Animal Ethics Committee of Laval University.

\section{OVA immunization}

Groups of C57BL/6 mice received 2 subcutaneous injections (day 1 and day 14) of $100 \mu \mathrm{g}$ of OVA emulsified in complete Freund adjuvant on day 1 and incomplete Freund adjuvant on day 14 (both from SigmaAldrich Canada, Oakville, ON, Canada). IVIg (Gamunex, Grifols Canada Ltd, Mississauga, Canada) was injected to the experimental group every day at $2.5 \mathrm{~g} / \mathrm{kg}$, starting two days before and ending two days after OVA injections, as previously described [1]. Control mice received the corresponding volume of vehicle (glycine $200 \mathrm{mM}, \mathrm{pH}$ 4.25). Mice were sacrificed 28 days after the first immunization. Spleens were recovered and homogenized with an organ grinder to obtain a single-cell suspension. Mouse plasma were collected and immediately stored at $-80^{\circ} \mathrm{C}$.

\section{Flow cytometry}

Cells were washed with PBS containing 1\% FBS (Fisher Scientific, Waltham, MA), centrifuged at $1000 \mathrm{~g}$ for 5 minutes and suspended in PBS containing 2\% FcR block (Miltenyi Biotec, Cambridge, MA) to minimize FcR-mediated $\mathrm{mAb}$ binding. The cell suspension was incubated 10 minutes at $4^{\circ} \mathrm{C}$ followed by addition of fluorescently labeled anti-mouse CD62L and anti-mouse CD8 (both from

*Corresponding author: Renée Bazin, Héma-Québec, Research and Development Department, 1070 Avenue des Sciences-de-la-Vie, Québec (Qc), Canada, Tel: 418-780-4362; E-mail: renee.bazin@hema-quebec.qc.ca

Received February 26, 2016; Accepted March 08, 2016; Published March 14 2016

Citation: Chabot D, Trépanier P, Ringuette-Goulet C, Loubaki L, Bazin R (2016) Role of the CD62L Expression Pathway in the IVIg Inhibition of Cytotoxic T Cell Responses. Immunochem Immunopathol 2: 117. doi: 10.4172/2469-9756.1000117

Copyright: $\odot 2016$ Chabot $D$, et al. This is an open-access article distributed under the terms of the Creative Commons Attribution License, which permits unrestricted use, distribution, and reproduction in any medium, provided the original author and source are credited. 
Citation: Chabot D, Trépanier P, Ringuette-Goulet C, Loubaki L, Bazin R (2016) Role of the CD62L Expression Pathway in the IVlg Inhibition of Cytotoxic T Cell Responses. Immunochem Immunopathol 2: 117. doi: 10.4172/2469-9756.1000117

eBioscience, San Diego, CA). The suspension was further incubated for 30 minutes at $4^{\circ} \mathrm{C}$ in the dark. Cells were then washed with PBS 1\% FBS and analyzed on a Partec Cyflow ML (Partec North American, Inc., Swedesboro, NJ) using FCS express 4 software (De NovoSoftware, Los Angeles, CA).

\section{ELISA}

For quantitation of sCD62L concentration, mouse plasma was collected at sacrifice and analyzed using an ELISA kit (mouse CD62L SELL ELISA Pair Set, Sino Biological Inc., Beijing, China) according to the manufacturer's instructions.

\section{Statistical analysis}

Results are presented as mean \pm standard error of the mean (SEM). All statistical analyses were performed using GraphPad InStat (GraphPad Software, La Jolla, CA) using appropriate tests. Values of $P<0.05$ were considered to indicate statistical significance.

\section{Results}

IVIg increases the surface expression of CD62L on splenic CD8+ T cells

To study the effect of IVIg on CD62L expression by T cells, we used a mouse model of OVA-immunization, in which the animals were treated or not with IVIg during the immunization period. We first examined the expression of CD62L on gated CD8+ T cells extracted from the spleen of OVA-immunized control (IVIg-) or IVIgtreated (IVIg+) mice (Figure 1A). The gate shown on the histogram was adjusted to delineate the different expression levels of CD62L (CD62 $\mathrm{L}^{\text {lo }}$ on the left and CD62 $\mathrm{L}^{\mathrm{hi}}$ on the right side). The results show that most CD8+ T cells from the control (IVIg-) group weakly express CD62L (mean MFI of $12.9 \pm 0.8, n=4$ ) on their surface (left side of the histogram). Similarly, a fraction of CD8+ T cells from IVIg-treated mice dose also weakly expressed CD62L (mean MFI of $15.8 \pm 3.8$, $\mathrm{n}=3)$. However, a significant proportion of CD8+ T cells $(37 \%)$ also expressed higher CD62L levels (mean MFI of $229.8 \pm 78.4$; right side of the histogram). The mean proportion of the CD62 $\mathrm{L}^{\text {hi }} \mathrm{CD} 8+\mathrm{T}$ cell population in control and IVIg-treated groups is shown in Figure 1B $(7.0 \pm 0.6 \%$ vs $30.0 \pm 6.5 \% ; P=0.002)$.

IVIg does not affect the surface expression of CD62L on splenic CD4+ T cells

We also examined the CD62L expression on splenic CD4+ T cells from control and IVIg-treated mice. In contrast to the observations done on CD8+ T cells, the analysis done on gated CD4+ T cells revealed the absence of a $\mathrm{CD} 62 \mathrm{~L}^{\text {hi }}$ cell population in IVIg-treated animals (Figure 2). Indeed, our results show a weak expression of CD62L on mouse CD4+ $\mathrm{T}$ cells recovered from the spleen of OVA-immunized mice in both control and IVIg-treated mice (left side of the histogram), indicating that the effect of IVIg on CD62L expression is restricted to CD8+ T cells in our model.

\section{IVIg treatment increases the concentration of $s C D 62 \mathrm{~L}$ in} mouse plasma

It is known that a soluble form of CD62L can be generated by cleavage of the surface bound CD62L. To determine whether IVIg could also affect the levels of sCD62L, we recovered the plasma of control and IVIg-treated mice at sacrifice and measured the sCD62L concentration by ELISA. As illustrated in Figure 3, we observed a significantly higher concentration of sCD62L compared to plasma from control animals
(A)

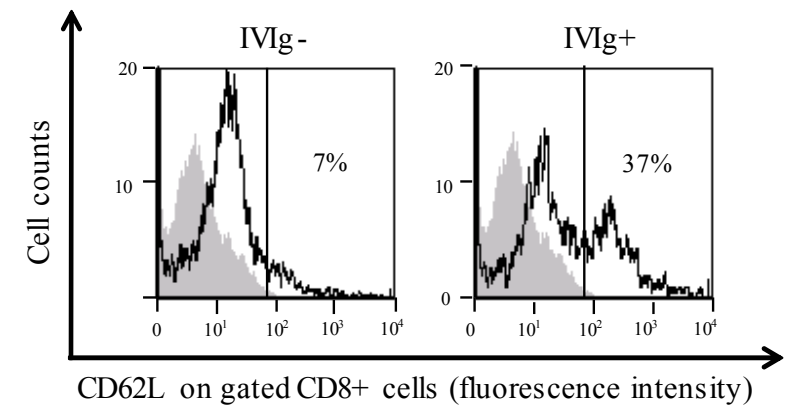

(B)

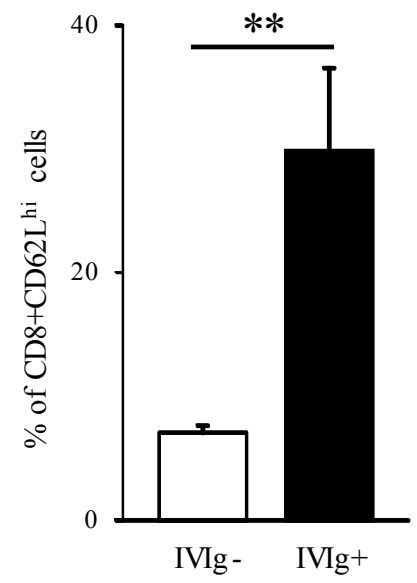

Figure 1: Effect of IVIg on the expression of CD62L on splenic CD8+ T cells. Spleens were recovered from control (IVIg-, $n=4)$ or IVlg-treated $(I V I g+, n=3$ ) mice 28 days after OVA immunization. Cells were stained with antibodies against CD8 and CD62L and analyzed by flow cytometry. The percentage of $C D 62 L^{\text {hi }}$ positive cells was evaluated on gated CD8+ T cells. (A) Results shown are representative of one of each group. The grey histogram represents unlabeled cells. The gate was set using the IVIg-treated cells to separate the two CD62L expression levels. (B) Mean percentage of CD8+CD62 ${ }^{\text {hi }}$ cells in control and IVlg-treated mice. ${ }^{* *} P<0.01$ (unpaired $t$ test)

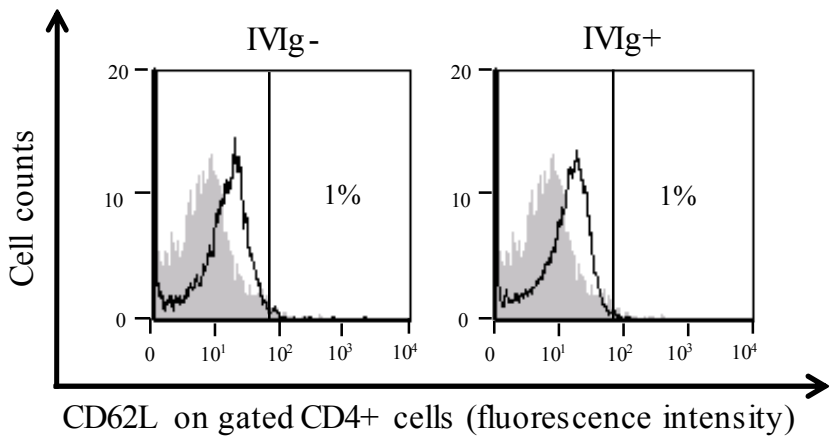

Figure 2: Effect of IVIg on the expression of CD62L on splenic CD4+ T cells. Spleens were recovered from control (IVlg-, $n=4)$ or IVIg-treated $(I V I g+, n=3$ ) mice 28 days after OVA immunization. Cells were stained with antibodies against CD4 and CD62L and analyzed by flow cytometry. CD62L expression was analyzed on gated CD4+ T cells recovered from the spleen of control (IVIg-, $\mathrm{n}=4$ ) or IVIg-treated (IVIg+, n=3 mice). Results shown are representative of one mouse from each group. 
Citation: Chabot D, Trépanier P, Ringuette-Goulet C, Loubaki L, Bazin R (2016) Role of the CD62L Expression Pathway in the IVIg Inhibition of Cytotoxic T Cell Responses. Immunochem Immunopathol 2: 117. doi: 10.4172/2469-9756.1000117

Page 3 of 4

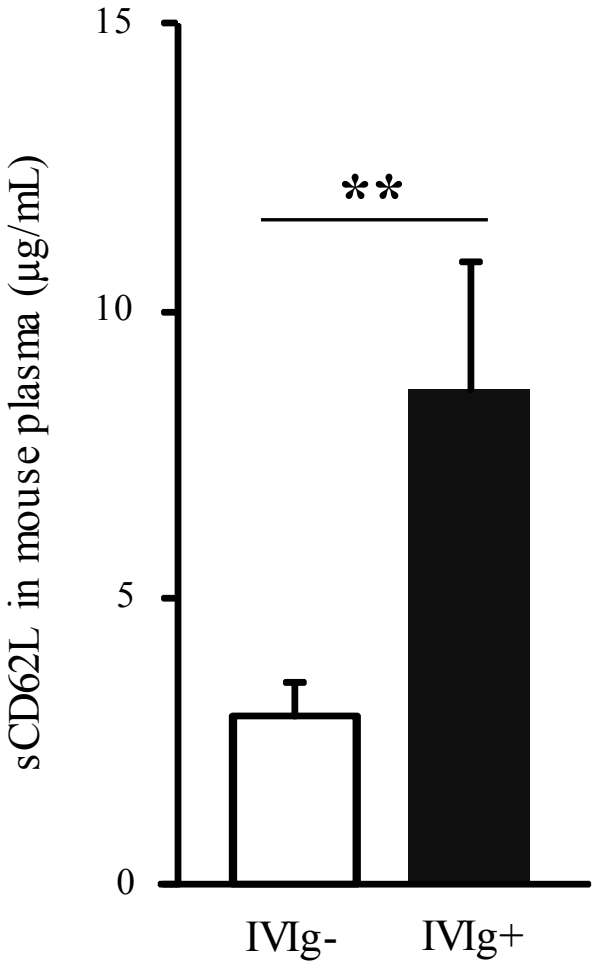

Figure 3: Effect of IVIg on SCD62L concentration in mouse plasma. The concentration of SCD62L in the plasma of control (IVIg-) or IVIg-treated mice ( $n=6$ mice per group) dose was determined by ELISA. Results are presented as mean \pm standard error of the mean. ${ }^{* \star} P<0.01$ (unpaired $t$ test).

$(8.6 \pm 2.2 \mu \mathrm{g} / \mathrm{mL}$ vs $3.0 \pm 0.6 \mu \mathrm{g} / \mathrm{mL} ; P<0.01)$, showing that the increased CD62L surface expression on CD8+ T cells is accompanied by a similar increase in plasma sCD62L.

\section{Discussion and Conclusion}

We recently reported that IVIg reduces cytotoxic responses in immunized C57BL/6 mice by decreasing the generation of OVAspecific CD8 $+\mathrm{T}$ cells as well as the proportion of CD8 $+\mathrm{T}$ cells expressing the cytotoxic-associated marker CD107a [1]. Because a direct correlation exists between the loss of CD62L expression on CD8+ T cells and the acquisition of both cytotoxic activity and surface expression of CD107a [6], we postulated that IVIg treatment during the OVA-immunization could result in a modulation of CD62L by CD8+ T cells. The results presented here support our hypothesis and further reveal an effect of IVIg on sCD62L levels that could also contribute to the anti-inflammatory effects of IVIg.

CTLs are the effector cells in cell-mediated immune responses and they are responsible of killing targeted cells by directed exocytosis of molecules like perforin and granzymes [8]. Degranulation by CTLs leading to the destruction of target cells requires actin remodelling to sustain the signal between CTLs and target cells [9-11]. As CD62L cytoplasmic tail is known to interact with the cytoskeleton [12], we suggest that the increased expression of CD62L on a significant proportion of CD8+ T cells in response to IVIg treatment may have an impact on the remodelling of the actin skeleton, leading to a reduced cytotoxic activity of CTLs.

Our results also revealed an increased concentration of sCD62L in the plasma of mice treated with $2.5 \mathrm{~g} / \mathrm{Kg}$ of IVIg. This higher level of sCD62L is likely to be related to CD62L shedding from the $\mathrm{CD} 62 \mathrm{~L}^{\text {hi }} \mathrm{CD} 8+\mathrm{T}$ cells. Whether this is due solely to the higher expression of CD62L or to an increase enzymatic activity of ADAM17 [13] remains to be determined. In a separate experiment, we determined that the concentration of sCD62L in mice treated with 1 $\mathrm{g} / \mathrm{Kg}$ remained similar to that of control animals (data not shown), suggesting that the level of CD62L on the surface of CD8+ T cells is not affected by IVIg at this concentration. This observation correlates with our previous observation that a dose of $1 \mathrm{~g} / \mathrm{Kg}$ was not sufficient to decrease the cytotoxic response in vivo, whereas a dose of $2.5 \mathrm{~g} / \mathrm{Kg}$ significantly reduced this response [1]. Interestingly, it has been shown that high levels of plasma sCD62L result in anti-inflammatory activity by inhibiting leukocyte migration and trafficking [7,14]. Indeed, CD62L-dependent leukocyte attachment was completely inhibited by sCD62L at concentrations ranging between 8 and $15 \mu \mathrm{g} / \mathrm{ml}$ [7]. Our results suggest that the concentration of sCD62L reached in the plasma of mice treated with high doses $(2.5 \mathrm{~g} / \mathrm{Kg})$ of IVIg could be sufficient to interfere with the ability of $\mathrm{T}$ cells to home to peripheral lymph nodes. Consequently, sCD62L may contribute to the decreased number of OVA-specific CTLs present in the spleen of IVIg-treated mice [1].

Finally, our results show that the expression of CD62L on CD4+ $\mathrm{T}$ cells is not affected in IVIg-treated mice. Interestingly, CD62Lexpressing CD4+ T cells have been associated with anti-inflammatory effects. More precisely, a CD62L+ subpopulation of CD4+ regulatory $\mathrm{T}$ cells was shown to protect from lethal acute graft versus host disease [15]. However, our results show that the effect of IVIg on CD62L surface expression on splenic T cells is restricted to CD8+ T cells in our model. The absence of CD62L modulation on CD4+ $\mathrm{T}$ cells by IVIg cannot be interpreted as an absence of IVIg effect on these cells; rather, this suggests that different mechanisms are involved in the IVIg effects on CD4+ and CD8+ T cell responses. Indeed, we and others have previously reported multiple anti-inflammatory effects of IVIg on CD4+ T cells (reviewed in Reference [16]). Nevertheless, we cannot rule out the possibility that such regulatory $\mathrm{CD} 62 \mathrm{~L}^{\text {hi }} \mathrm{CD} 4+\mathrm{T}$ cells are induced by IVIg treatment since we have analyzed only splenic and not peripheral blood $\mathrm{T}$ cells.

Although the above results were obtained in mice, it is tempting to speculate that the CD62L pathway could be involved in the therapeutic effects observed in IVIg-treated patient suffering from inflammatory disorders involving CTLs, such as Crohn's disease [17], toxic epidermal necrolysis [18], type 1 diabetes [19] and many neurological disorders [20]. Interestingly, a lower expression of CD62L was observed on $\mathrm{CD} 3+\mathrm{T}$ cells from type 1 diabetes patients compared to healthy counterparts [21], supporting the above possibility. Overall, our results support a role for the CD62L expression pathway of CD8+ T cells in the anti-inflammatory effects of IVIg and broaden our understanding of its complex mechanisms of action in autoimmune and inflammatory disorders.

\section{Acknowledgments}

We thank all the volunteers for their participation in this study. DC is recipient of an Industrial Innovation Scholarship from the National Sciences and Engineering Research Council of Canada (NSERC) and Fonds Québécois de Recherche Nature et Technologies (FQRNT).

\section{References}

1. Trépanier $P$, Chabot $D$, Bazin $R$ (2014) Intravenous immunoglobulin modulates the expansion and cytotoxicity of CD8+ T cells. Immunology 141: 233-241.

2. Aktas E, Kucuksezer UC, Bilgic S, Erten G, Deniz G (2009) Relationship between CD107a expression and cytotoxic activity. Cell Immunol 254: 149-154. 
Citation: Chabot D, Trépanier P, Ringuette-Goulet C, Loubaki L, Bazin R (2016) Role of the CD62L Expression Pathway in the IVIg Inhibition of Cytotoxic T Cell Responses. Immunochem Immunopathol 2: 117. doi: 10.4172/2469-9756.1000117

3. Warnock RA, Askari S, Butcher EC, von Andrian UH (1998) Molecular mechanisms of lymphocyte homing to peripheral lymph nodes. J Exp Med 187 205-216.

4. Arbonés ML, Ord DC, Ley K, Ratech H, Maynard-Curry C, et al. (1994) Lymphocyte homing and leukocyte rolling and migration are impaired in L-selectin-deficient mice. Immunity 1: 247-260.

5. Tedder TF, Penta AC, Levine HB, Freedman AS (1990) Expression of the human leukocyte adhesion molecule, LAM1. Identity with the TQ1 and Leu-8 differentiation antigens. J Immunol 144: 532-540.

6. Yang S, Liu F, Wang QJ, Rosenberg SA, Morgan RA (2011) The shedding of CD62L (L-selectin) regulates the acquisition of lytic activity in human tumor reactive T lymphocytes. PLoS One 6: e22560.

7. Schleiffenbaum B, Spertini O, Tedder TF (1992) Soluble L-selectin is presen in human plasma at high levels and retains functional activity. J Cell Biol 119 229-238.

8. Trapani JA, Smyth MJ (2002) Functional significance of the perforin/granzyme cell death pathway. Nat Rev Immunol 2: 735-747.

9. Shen A, Puente LG, Ostergaard HL (2005) Tyrosine kinase activity and remodelling of the actin cytoskeleton are co-temporally required for degranulation by cytotoxic T lymphocytes. Immunology 116: 276-86.

10. Stinchcombe JC, Majorovits E, Bossi G, Fuller S, Griffiths GM (2006) Centrosome polarization delivers secretory granules to the immunological synapse. Nature 443: 462-465.

11. Dustin ML, Cooper JA (2000) The immunological synapse and the actin cytoskeleton: molecular hardware for T cell signaling. Nat Immunol 1: 23-29.

12. Pavalko FM, Walker DM, Graham L, Goheen M, Doerschuk CM, et al. (1995) The cytoplasmic domain of L-selectin interacts with cytoskeletal proteins via alpha-actinin: receptor positioning in microvilli does not require interaction with alpha-actinin. J Cell Biol 129: 1155-1164.
13. Li Y, Brazzell J, Herrera A, Walcheck B (2006) ADAM17 deficiency by mature neutrophils has differential effects on L-selectin shedding. Blood 108: 22752279

14. Ferri LE, Swartz D, Christou NV (2001) Soluble L-selectin at levels present in septic patients diminishes leukocyte-endothelial cell interactions in mice in vivo: a mechanism for decreased leukocyte delivery to remote sites in sepsis. Crit Care Med 29: 117-122.

15. Ermann J, Hoffmann P, Edinger M, Dutt S, Blankenberg FG, et al. (2005) Only the $\mathrm{CD} 62 \mathrm{~L}+$ subpopulation of $\mathrm{CD} 4+\mathrm{CD} 25+$ regulatory $\mathrm{T}$ cells protects from lethal acute GVHD. Blood 105: 2220-2226.

16. Padet L, Bazin R (2014) Effects of IVlg on T Cell Functions. Plasmapheresis and Intravenous Immunoglobin: Clinical Uses. Potential Complications and Long-Term Health Effects. pp: 107-135.

17. Körber J, Köttgen E, Renz H (1998) A case of Crohn's disease with increased CD8 T-cell activation and remission during therapy with intravenous immunoglobulins. Scand J Gastroenterol 33: 1113-1117.

18. Trent JT, Kirsner RS, Romanelli P, Kerdel FA (2003) Analysis of intravenous immunoglobulin for the treatment of toxic epidermal necrolysis using SCORTEN: The University of Miami Experience. Arch Dermatol 139: 39-43.

19. Bulek AM, Cole DK, Skowera A, Dolton G, Gras S, et al. (2012) Structural basis for the killing of human beta cells by $\mathrm{CD} 8(+) \mathrm{T}$ cells in type 1 diabetes. Nat Immunol 13: 283-289.

20. Deckert M, Sanchez-Ruiz M, Brunn A, Schluter D (2010) Role of CD8 T-cellmediated autoimmune diseases of the central nervous system. Crit Rev Immunol 30: 311-326.

21. MacKinnon JR, Knott RM, Forrester JV (2004) Altered L-selectin expression in lymphocytes and increased adhesion to endothelium in patients with diabetic retinopathy. $\mathrm{Br} \mathrm{J}$ Ophthalmol 88: 1137-1141. 